\title{
Stable vector bundles on the families of curves
}

\author{
Fedor A. Bogomolov ${ }^{1,2}$. Elena Lukzen ${ }^{3}$
}

Received: 30 October 2020 / Revised: 20 December 2021 / Accepted: 23 December 2021 /

Published online: 14 February 2022

(c) The Author(s) 2022

\begin{abstract}
We offer a new approach to proving the Chen-Donaldson-Sun theorem which we demonstrate with a series of examples. We discuss the existence of a construction of a special metric on stable vector bundles over the surfaces formed by a family of curves and its relation to the one-dimensional cycles in the moduli space of stable bundles on curves.
\end{abstract}

Keywords Vector bundle $\cdot$ Stability $\cdot$ Families of curves

Mathematics Subject Classification 14D20 $\cdot 14 \mathrm{H} 10 \cdot 14 \mathrm{~J} 60$

\section{Introduction}

Stability of vector bundles on different geometric spaces has been an object of study for a long time deriving from work in algebraic geometry. The concept of stability arose naturally from the question of whether a space which could parameterize all bundles of a given rank exists, moreover, a subsequent question was if there is a special class of vector bundles which could be naturally parametrized in the same way. Significant progress in the direction has been made by Mumford [19,20], Maruyama [16-18],

The first author was partially supported by the HSE University Basic Research Program and by EPSRC programme grant EP/M024830. The second author is supported by the SISSA PhD fellowship and acknowledges the support of the H2020-MSCA-RISE-2017 PROJECT No. 778010 IPADEGAN.

Elena Lukzen

elukzen@sissa.it

Fedor A. Bogomolov

bogomolo@cims.nyu.edu

1 Courant Institute, New York Universuty, 251 Mercer Str., New York, NY 10012, USA

2 Laboratory of Algebraic Geometry, Department of Mathematics, The National Research University Higher School of Economics, 6 Usacheva Str., Moscow, Russia 119048

3 SISSA, Via Bonomea 265, 34136 Trieste, Italy 
Narasimhan-Seshadri [21], Yau [25], Donaldson, Uhlenbeck-Yau [12,13,24], Hitchin [14], Corlette [10], Simpson [23] and others. The Donaldson-Uhlenbeck-Yau theorem relates Hermitian-Yang-Mills connections to an algebraic property of the holomorphic vector bundle of being stable.

There are several results concerning the existence of special metrics on stable bundles over complex projective manifolds. One of the first improvements in this question was made by Ruadhaí Dervan and Julius Ross, see [11] for more details. One of the recent ones is the Chen-Donaldson-Sun theorem [7-9] which claims the existence of a flat unitary metric on a stable bundle with Chern classes $c_{1}, c_{2}$ equal zero. It was obtained by considering a special Futaki-type functional on the space of Hermitian metrics and proving the existence of a metric with a minimal value of the functional. The resulting metric happens to be flat in case of $c_{2} \geqslant 0$ which implied the result, see [7-9] for more details.

Recall that all Kähler metrics in the class $[\omega]$ can be parametrized by smooth functions in the space of Kähler potentials $\mathcal{H}_{\omega}=\left\{\varphi \in C^{\infty}(M): \omega_{\varphi}=\omega+\sqrt{-1} \partial \bar{\partial} \varphi>0\right\}$. Then, for $\varphi \in \mathcal{H}_{\omega}$, the scalar curvature is $R_{\varphi}=-g_{\varphi}^{i \bar{j}} \partial_{i} \partial_{\bar{j}} \log \left(\operatorname{det}\left(g_{i \bar{j}}+\varphi_{i \bar{j}}\right)\right)$ and the constant scalar curvature equation reads as $R_{\varphi}=R^{\prime}$, where $R^{\prime}$ is a constant depending only on the manifold, class and complex structure $(M,[\omega], J)$.

Conjecture 1.1 (Yau-Tian-Donaldson Conjecture) $(X, L)$ admits a CSCK metric if and only if it is $K$-stable.

The YTD conjecture is proven for Fano and tropical varieties. In this article, we show how one-dimensional cycles in the moduli space of vector bundles on curves are associated to bundles on families of curves which are stable on the restriction to every curve of the family. We present a connection between existence of a special metric on a class of surfaces formed by the families of curves and its' natural relation to one-dimensional cycles in the moduli space of stable bundles on curves.

Suppose that $B$ is a family of curves, $B=\left\{C_{\lambda}\right\}_{\lambda \in \Lambda}$, where $\Lambda$ is a parameter set. Assume that the vector bundle $E$ on $B$ has a stable restriction on any curve $C_{\lambda}$. If, in addition, the first Chern class of the restriction $E_{\lambda}$ of $E$ on any curve $C_{\lambda}$ is trivial, then by [21] there is a flat Hermitian metric $h_{i, j}$ on $E_{\lambda}$ which is unique modulo multiplication by a constant. Thus, it defines the unique flat Hermitian connection on $E_{\lambda}$ and the unique modulo conjugation irreducible unitary representation $\rho_{\lambda}: \pi_{1}\left(C_{\lambda}\right) \rightarrow U(n)$.

The choice of the flat Hermitian metric $h_{i, j}$ on $E_{\lambda}$ for any $\lambda \in B$ defines a Hermitian metric $h$ on $E$ over $B$. The metric $h$ is not unique, but depends on a real-valued invertible function of $\Lambda$ corresponding to the choice of the actual Hermitian metric on $E_{\lambda}$. Hence we obtain a family of such metrics on $E$ and corresponding Hermitian connections $\Theta_{h}$. The latter defines Chern forms representing all Chern classes of $E$. We are particularly interested in the local properties of the $(2,2)$-form on $B$ representing the second Chern class $c_{2}(E)$ obtained from $\Theta_{h}$ when $B$ is a two-dimensional complex surface. We can express the second Chern class via the curvature of the cycle $\mathcal{S}$ in the moduli space of vector bundles, which corresponds to a particular vector bundle $E$ on the base $B$ :

$$
c_{2}(E)=\frac{\operatorname{tr}\left(\Theta(\mathcal{S})^{2}\right)-\operatorname{tr}^{2}(\Theta(\mathcal{S}))}{8 \pi^{2}} .
$$


The following result can be obtained by a direct calculation (see Sects. 6 and 7).

Theorem 1.2 The second Chern class is represented locally by the formula

$$
c_{2}(E)=\sum_{i, j}-\frac{\operatorname{det}\left(D_{i j}\right) \operatorname{det}\left(\bar{D}_{i j}\right)}{\operatorname{det}\left(h_{i j}\right)} d z^{1} \wedge d \bar{z}^{1} \wedge d z^{2} \wedge d \bar{z}^{2}
$$

Corollary 1.3 The form representing the second Chern class $c_{2}(E)$ on $B$ is nonnegative, since $\frac{\operatorname{det}\left(D_{i j}\right) \operatorname{det}\left(\bar{D}_{i j}\right)}{\operatorname{det}\left(h_{i j}\right)} \geqslant 0$.

Corollary 1.4 Assume that (- $\left.\operatorname{det} \partial^{2} h_{i, j}\right)$ is nonzero somewhere on $B$, then $c_{2}(E)$ is semipositive. In particular, if the base $\Lambda$ is a compact curve and the representation $\rho_{\lambda}$ varies modulo conjugation on $\Lambda$, then $c_{2}(E)$ has a representative which is a semipositive $(2,2)$-form.

This fact yields a possibility for a constructive approach to the proof of the ChenDonaldson-Sun theorem for a projective surface $X$. The theorem claims that $c_{2}(E)>$ 0 for a stable vector bundle with $c_{1}(E)=0$ unless it is induced from a unitary representation of $\pi_{1}(B)$. Our explicit local calculation implies this result for a compact surface $f: B \rightarrow C$ fibered with all smooth fibres $f^{-1}(c), c \in C$, over a smooth curve and a bundle $E$ which has stable restrictions on the fibers $f^{-1}(c)$.

Remark 1.5 We should note that Theorem 1.2 holds not only for stable bundles $E \rightarrow B$ on our class of surfaces (see [6] for more details). In particular, if the bundle $E$ is not stable, but restricts to a stable one to a curve $C \subset B$, this result holds as well.

We also discuss other possible applications and generalizations of the above approach.

We should note that several results in a similar direction have recently appeared on arXiv [1,2,5]. We should also mention the work of O'Grady [22] in a similar direction.

\section{Preliminary facts and background}

\subsection{Bogomolov's restriction theorem, cones of polarizations and effective divisors}

Let us recall a number of facts following the exposition presented in the book [15]. In the case of smooth projective surfaces we have a nondegenerate hyperbolic scalar product on the group $\operatorname{Pic}_{R} V$ and duality between the cone of polarizations and the cone of effective divisors.

Let Num denote the free $\mathbf{Z}$-module Pic $(X)$ factorized by the numerical equivalence. The intersection product defines an integral quadratic form on Num.

The Hodge Index Theorem says, that, over $\mathbf{R}$, the positive definite part is onedimensional. In other words, Num $_{\mathbf{R}}$ carries the Minkowski metric. For any class $u \in$ $\operatorname{Num}_{\mathbf{R}}$ let $|u|=\left|u^{2}\right|^{1 / 2}$.

Denote as $A$ a subcone consisting of all ample divisors. A polarization of $X$ is a ray $\mathbf{R}_{>0} . H$, where $H \in A$. Let $\mathcal{H}$ denote the set of rays in $K_{+}$. This set can be identified with the hyperbolic manifold $\left\{H \in K_{+}:|H|=1\right\}$. 
The hyperbolic metric $\beta$ is defined as follows: for points $[H],\left[H^{\prime}\right] \in \mathcal{H}$ let

$$
\beta\left([H],\left[H^{\prime}\right]\right)=\operatorname{arccosh} \frac{H \cdot H^{\prime}}{|H| .\left|H^{\prime}\right|} .
$$

Denote the open cone $K^{+}=\left\{D \in \operatorname{Num}_{R}: D^{2}>0, D . H>0\right.$ for all ample divisors $H\}$. Note that the second condition is added only to pick one of the two connected components of the set of all $D$ with $D^{2}>0$. This cone contains the cone of ample divisors and in turn is contained in the cone of effective divisors. $K^{+}$satisfies the following property: $D \in K^{+}$iff $D . L>0$ for all $L \in \overline{K^{+}} \backslash\{0\}$.

For any pair of sheaves $G, G^{\prime}$ with nonzero rank let

$$
\xi_{G^{\prime}, G}:=\frac{c_{1}\left(G^{\prime}\right)}{r k\left(G^{\prime}\right)}-\frac{c_{1}(G)}{r k(G)} \in \operatorname{Num}_{R}
$$

This invariant in the case of a destabilizing sheaf plays the role of a "destabilizing" point in the corresponding cone.

Theorem 2.1 Let $X$ be a smooth projective variety of dimension $n$ and $H$ an ample divisor on $X$. If $F$ is a semistable torsion free sheaf, then

$$
\Delta(F) \cdot H^{n-1} \geqslant 0 .
$$

Theorem 2.2 (Bogomolov's effective restriction '78 [3,4]) Let $W$ be a locally free sheaf of rank $r \geqslant 2$ on an algebraic surface with $c_{1}(W)=0$. Assume that $W$ is $\mu$-stable with respect to an ample class $H \in K^{+} \cap$ Num and $C \subset X$ be a smooth curve with $[C]=n H$. Let $2 n \geqslant \frac{R}{r} \Delta(F)+1$. Then $\left.F\right|_{C}$ is a stable sheaf.

A consequence of the theorem is that depending on $c_{1}$ and rank, the restriction of a vector bundle $E$ to curves (in particular to the ones with a relatively small number of singularities) is stable.

\section{Moduli section}

Let us introduce a new object arising from every vector bundle on particular families. Suppose we have $B$, a family of curves, and $\mathcal{E}$, a vector bundle on it. We can say that $\left.\mathcal{E}\right|_{C_{\lambda}}$ restricted to a point of the family, i.e. a curve $C_{\lambda}$, is a bundle on a Riemann surface with the corresponding unitary connection $\nabla$. The information on stability of $\mathcal{E}$ is contained in its second Chern class $c_{2}(\mathcal{E})$.

Definition 3.1 Every vector bundle $\mathcal{E}$ on $B$, which is stable on the restriction to any curve of the family $B$, is called a smooth section of the family of moduli spaces $M_{C_{\lambda}}$, $\lambda \in \Lambda$,

$$
\mathcal{E} \in \Gamma\left(M_{C_{\lambda}}\right)_{\lambda \in \Lambda}
$$


Motivation for the definition. The vector bundle $\mathcal{E}$ on the family of curves $B$ on the restriction to the point $\left.\mathcal{E}\right|_{C_{\lambda_{0}}}$, i.e. a particular curve $C_{\lambda_{0}}$, is a vector bundle, arising from the representation of the fundamental group of $\pi_{1}\left(C_{\lambda_{0}}\right)$ (by the Narasimhan-Seshadri theorem). Therefore we can simply consider the following fibration on $B$ : the fiber on every curve $C_{\lambda}$ corresponds to its moduli space $M_{C_{\lambda}}$. Consequently, to "fix a vector bundle on the family $B$ " simply means that on each fiber, i.e. on the corresponding moduli space, we have to choose one element. It identically repeats the definition.

To supplement our definition we should note that stable bundles come from:

- Stable bundles on normalizations of the singular curves: $\mathcal{E} \rightarrow \widehat{C}$, where $v: \widehat{C} \rightarrow C$ is a normalization. One can get these bundles from representations of a fundamental group of the normalization $\pi_{1}(\widehat{C})$ and thus the destabilizing sheaf is not induced on the initial curve $C$.

- Bundles which are unstable on the normalization $\widehat{C}$, but are stable on the singular curve $C$ (parabolic semistable vector bundles). They appear in the following way: we can take an unstable bundle $\widehat{\mathcal{E}}$ (i.e. with destabilizing subsheaf $\mathcal{F}$ ) on the normalization $\widehat{C}$. When we arrive to a base $C$ it transforms to a point $\mathcal{E}^{\text {sing }}$. In such a way we obtain an identification of the parameters of the destabilizing sheaf $\mathcal{F}$ with the structure of the parabolic bundle on $C$. It means that on $\widehat{\mathcal{E}} \rightarrow \widehat{X}$, where $\widehat{\mathcal{E}}$ is an unstable bundle at the point on the normalization $\widehat{X}(\widehat{X}$ denotes $X$ blown up in a node of a singular curve $C, v: \widehat{C} \rightarrow C$ ). Then it has a normal destabilizing subsheaf $\mathcal{F} \subset \widehat{\mathcal{E}}$ such that $c_{1}(\mathcal{F})-\left(\frac{\text { rk } \mathcal{F}}{k}\right) c_{1}(\mathcal{E}) \in K^{+}$.

A blow-down map $\hat{v}$ induces a morphism

$$
\hat{v}: \mathcal{F} \rightarrow \mathcal{E}_{p},
$$

where $\mathcal{E}_{p}$ is a flag variety of type determined by a fixed quasiparabolic structure, i.e. a flag $\mathcal{E}_{p}=F_{1} \mathcal{E}_{p} \supset F_{2} \mathcal{E}_{p} \supset \cdots \supset F_{r} \mathcal{E}_{p}$ and weights $\alpha_{1}, \ldots, \alpha_{r}$ attached to $F_{1} \mathcal{E}_{p}, \ldots, F_{r} \mathcal{E}_{P}$ such that $0 \leqslant \alpha_{1}<\alpha_{2}<\cdots<\alpha_{r}<1, k_{1}=\operatorname{dim} F_{1} \mathcal{E}_{p}-$ $\operatorname{dim} F_{2} \mathcal{E}_{p}, k_{r}=\operatorname{dim} F_{r} \mathcal{E}_{P}$ the multiplicities of $\alpha_{1}, \ldots, \alpha_{r}$.

These are parabolic bundles. Thus we get a strata, which is glued to our families of moduli spaces of vector bundles. It is known that the set of all parabolic semistable bundles is an open subset of a suitable Hilbert scheme, which has the usual properties, i.e. non-singular, irreducible and of a given dimension. This open set could be mapped to a product of Grassmannians and flag varieties.

Remark 3.2 If $C_{\lambda}=C$ is a constant curve, then all the moduli spaces are the same $M_{C_{\lambda}}=M_{C}=M$, thus every vector bundle on $B$ is decoded by a subset $\mathcal{S} \subset M$ in the moduli space $M$ :

$$
\mathcal{S}=\left\{m_{\lambda} \in M: \lambda \in \Lambda\right\}
$$

Remark 3.3 The moduli space $M_{B}$ of all the vector bundles on $B$ consists of all the sections $S$ of the family of the corresponding moduli spaces $M_{C_{\lambda}}$.

Remark 3.4 If $C_{\lambda}=C_{1}$ and $\Lambda=C_{2}$, we have a vector bundle on $C_{1} \times C_{2}$. Then every subset $\mathcal{C}_{1} \subset M_{C_{2}}$ or $\mathcal{C}_{2} \subset M_{C_{1}}$ will form a cycle in the corresponding moduli space of vector bundles. 
This way we have a correspondence between families of stable vector bundles and the sections of families of moduli spaces of vector bundles over a given base.

\section{Ehresmann-induced connection for a smooth family of curves}

Let $\mathcal{E} \rightarrow B$ be a vector bundle such that $\left.\mathcal{E}\right|_{f^{-1}(\lambda)}$ for $\lambda \in C$. Coordinates on $\mathcal{E} \rightarrow$ $B$ could be described as a pair of representations $\left(\tau_{\lambda}, \rho\right)$, where $\tau_{\lambda}$ corresponds to a horizontal bundle and $\rho$ corresponds to a vertical bundle. The fiber is equal to $\left.\mathcal{E}\right|_{(x, y)}=E_{\rho} \cap E_{\tau_{\lambda}}=\left.E\right|_{\left(\rho, \tau_{\lambda}\right)}$.

As is well known, along $E_{\rho}, E_{\tau_{\lambda}}$ there exist Ehresmann connections which can be viewed as horizontal subbundles $H_{\rho}, H_{\tau}$ :

$$
T E_{\rho}=H_{\rho} \oplus V_{\rho}, \quad T E_{\tau_{\lambda}}=H_{\tau_{\lambda}} \oplus V_{\tau_{\lambda}}
$$

Denote $\mathcal{C}_{1}:=\left\{C \subset\left(\mathcal{M}_{C_{\lambda}}\right)_{\lambda \in C}\right\}$ and $\mathcal{C}_{2}:=\left\{\left(C_{\lambda}\right)_{\lambda \in C} \subset \mathcal{M}_{C}\right\}$. Thus we have two families of Ehresmann connections $\left\{H_{\rho}: \rho \in \mathcal{C}_{1}\right\}$ and $\left\{H_{\tau}: \tau \in \mathcal{C}_{2}\right\}$.

We are seeking for the connection $H$ on the bundle $\mathcal{E}$ such that $T \mathcal{E}=H \oplus V$, where $V$ is a tangent space to a fiber. Note that $V=T\left(E_{\rho} \cap E_{\tau_{\lambda}}\right)=\left(H_{\rho} \oplus V_{\rho}\right) \cap\left(H_{\tau_{\lambda}} \oplus V_{\tau_{\lambda}}\right)$.

The tangent to $T \mathcal{E}$ is a tangent bundle of a moduli cycle $\mathcal{C}_{1}$. Locally $T \mathcal{E}=$ $T E_{\rho} \oplus T E_{\tau_{\lambda}}$, therefore we can define a possible connection at least set-theoretically:

$$
H=\left(H_{\rho} \oplus V_{\rho}\right) \oplus\left(H_{\tau_{\lambda}} \oplus V_{\tau_{\lambda}}\right) /\left\{\left(H_{\rho} \oplus V_{\rho}\right) \cap\left(H_{\tau_{\lambda}} \oplus V_{\tau_{\lambda}}\right)\right\} .
$$

Definition 4.1 We call a connection $\nabla_{\mathcal{E}}$ induced from flat connections from both directions the Ehresmann-induced connection (EIC, for short).

Theorem 4.2 Denote by $\Theta_{\mathcal{E}}$ a curvature of connection $\nabla_{\mathcal{E}}$. The obstruction to the Jordan property - failure for the tangent fields $V, W$ to the connection to generate a Lie algebra - is exactly the curvature $\Theta_{\mathcal{E}}$.

Proof Our goal is to find an expression for the curvature tensor $\Theta$ of the connection on the vector bundle $\mathcal{E}$. Therefore, we would be able to express the second Chern class $c_{2}(\mathcal{E})$ explicitly, depending on a moduli cycle $\mathcal{S}$ :

$$
c_{2}(\mathcal{E})=\frac{\operatorname{tr}\left(\Theta(\mathcal{S})^{2}\right)-\operatorname{tr}^{2}(\Theta(\mathcal{S}))}{8 \pi^{2}} .
$$

Notice that there exist only two "directions" on the base space $B$ : a direction along a curve $C_{\lambda}$ and a direction along a curve $C$, which parameterizes the family. When we lift vector fields from the base $B$ to a vector bundle, we get the vector fields $V, W$ along the "cycle/section" $\mathcal{C}_{1}$ and along the curve $C . V$ and $W$ induce diffeomorphisms $\varphi_{V}, \psi_{W}$ on a vector bundle $\mathcal{E}$, which act on particular sections $s \in \Gamma(\mathcal{E})$. If we differentiate the result twice $\partial^{2} \varphi_{V} \psi_{W}^{-1} \varphi_{V}^{-1} \psi_{W}(s)$, we will get $\Theta(s)$ a value of curvature on $s$. If we take a corresponding basis of sections of $\mathcal{E}$ and lift the basis of vector fields $V, W$ from the base to our cycle $C$ in the moduli space of vector bundles and look how do the diffeomorphisms act on the basis of sections, we would be able to count $\Theta_{\mathcal{E}}$ explicitly. 
As is well known in this case, the obstruction to the Jordan property - failure for the tangent fields $V, W$ to the connection to generate a Lie algebra - is the curvature $\Theta_{\mathcal{E}}$.

Note that we can use Gauss-Manin connection which acts as $\nabla_{\mathrm{GM}}: T \mathcal{C} \rightarrow V$ (by the reason that $\nabla_{\mathrm{GM}}$ is flat), where $V$, as above, is a tangent space to a fiber.

Recall that by non-Abelian Hodge theory, the tangent space at the point $(C, U)$ which is equal to $M_{\mathrm{dR}}\left(C_{u}\right)$ is canonically identified with $H_{\mathrm{dR}}^{1}\left(C_{u}\right.$, End $\left.E\right)$.

Therefore a Gauss-Manin connection computes the derivative of our section $S$. Notice that we have not only the Gauss-Manin connection, but also the other one induced from the connections from the both directions whose properties we will study in the following sections.

\section{Calculation of the second Chern class}

Recall that the Dolbeaut representative of the Atiyah class is given by $[\bar{\partial}, \Omega]$, where $\Omega$ is a curvature tensor on the vector bundle $E$. In coordinates,

$$
\Omega_{j}^{i}=\sum R_{j \alpha \bar{\beta}}^{i} d z^{\alpha} \wedge d \bar{z}^{\beta}
$$

therefore, for $C=\Omega \wedge \Omega=\sum_{k}\left(\Omega_{k}^{i} \wedge \Omega_{j}^{k}\right)$. As every $\Omega_{i j}$ has a form $\Omega_{j}^{i}=$ $\sum R_{j \alpha \bar{\beta}}^{i} d z^{\alpha} \wedge d \bar{z}^{\beta}$, one has

$$
\begin{aligned}
C_{i j} & =\sum \Omega_{k}^{i} \wedge \Omega_{j}^{k}=\sum R_{k \alpha \bar{\beta}}^{i} d z^{\alpha} \wedge d \bar{z}^{\beta} \wedge \sum R_{j \alpha \bar{\beta}}^{k} d z^{\alpha} \wedge d \bar{z}^{\beta} \\
& =\sum_{k} \sum_{\alpha, \beta, \gamma, \theta} \sum_{\sigma \in S_{4}}(-1)^{\sigma} R_{k \sigma(\alpha \bar{\beta})}^{i} R_{j \sigma(\gamma \bar{\theta})}^{k} d z^{\alpha} \wedge d z^{\beta} \wedge d \bar{z}^{\gamma} \wedge d \bar{z}^{\theta}
\end{aligned}
$$

Denote by $(\alpha)=(\alpha, \beta, \gamma, \theta)$ a multindex consisting of four variables. Then,

$$
\begin{aligned}
\left(\Omega^{4}\right)_{i i}= & \sum_{j}\left(\Omega^{2}\right)_{i j} \wedge\left(\Omega^{2}\right)_{j i} \\
= & \sum_{j} \sum_{(\alpha)}\left[\sum_{k} \sum_{\sigma \in S_{4}}(-1)^{\sigma} R_{k \sigma^{\prime} \cdot \sigma(\alpha \bar{\beta})}^{i} R_{j \sigma^{\prime} \cdot \sigma(\gamma \bar{\theta})}^{k}\right] \\
& \cdot\left[\sum_{k} \sum_{\sigma \in S_{4}}(-1)^{\sigma} R_{k \sigma^{\prime} \cdot \sigma\left(\alpha^{\prime} \bar{\beta}^{\prime}\right)}^{j} R_{i \sigma^{\prime} \cdot \sigma\left(\gamma^{\prime} \bar{\theta}^{\prime}\right)}^{k}\right] d z^{\alpha} \wedge d z^{\beta} \wedge d \bar{z}^{\gamma} \wedge d \bar{z}^{\theta} .
\end{aligned}
$$

Recall that

$$
R_{j \bar{k} \alpha \bar{\beta}}=\sum h_{i \bar{k}} R_{j \alpha \bar{\beta}}^{i}=-\partial_{\bar{\beta}} \partial_{\alpha} h_{j \bar{k}}+\sum h^{a \bar{b}} \partial_{\alpha} h_{j \bar{b}} \partial_{\bar{\beta}} h_{a \bar{k}}
$$


Recall that $\left(h^{\beta \gamma}\right)=h^{-1}$ and

$$
R_{i \bar{j} \alpha}^{\beta}=-h^{\beta \gamma}\left(\partial^{2} \frac{h_{\alpha \bar{\gamma}}}{\partial z^{i} \partial \bar{z}^{j}}-h^{\delta \mu} \frac{\partial h_{\alpha \mu}}{\partial z^{i}} \frac{\partial h_{\delta \bar{\gamma}}}{\partial \bar{z}^{j}}\right)
$$

and the curvature

$$
\Omega=\sum R_{i \bar{j} \alpha}^{\beta} d z^{i} \wedge d \bar{z}^{j} \otimes e^{* \alpha} \otimes e_{\beta}
$$

For our expression we would need

$$
R_{\sigma^{\prime}(k) \cdot \sigma(\alpha \bar{\beta})}^{j}=h^{j k}\left(-\partial_{\sigma^{\prime} \cdot \sigma(\bar{\beta})} \partial_{\sigma^{\prime} \cdot \sigma(\alpha)} h_{i \bar{k}}+\sum h^{a \bar{b}} \partial_{\sigma^{\prime} \cdot \sigma(\alpha)} h_{i \bar{b}} \partial_{\sigma^{\prime} \cdot \sigma \beta} h_{a \bar{k}}\right) .
$$

Then we will have

$$
\begin{aligned}
\left(\Omega^{4}\right)_{i i}=\sum_{j} \sum_{\alpha}\left[\sum_{k} \sum_{\sigma \in S_{4}}(-1)^{\sigma} h^{i k} h^{k j} h^{j k} h^{k i}\right. \\
\cdot\left(-\partial_{\sigma(\bar{\beta})} \partial_{\sigma(\alpha)} h_{i \bar{k}}+\sum h^{a \bar{b}} \partial_{\sigma(\alpha)} h_{i \bar{b}} \partial_{\sigma \beta} h_{a \bar{k}}\right) \\
\left.\cdot\left(-\partial_{\sigma(\bar{\theta})} \partial_{\sigma(\gamma)} h_{k \bar{j}}+\sum h^{a \bar{b}} \partial_{\sigma(\gamma)} h_{k \bar{b}} \partial_{\sigma \theta} h_{a \bar{j}}\right)\right] \\
\cdot\left[\sum_{k} \sum_{\sigma \in S_{4}}(-1)^{\sigma}\left(-\partial_{\sigma(\bar{\beta})} \partial_{\sigma(\alpha)} h_{j \bar{k}}+\sum h^{a \bar{b}} \partial_{\sigma(\alpha)} h_{j \bar{b}} \partial_{\sigma \beta} h_{a \bar{k}}\right)\right. \\
\left.\cdot\left(-\partial_{\sigma(\bar{\theta})} \partial_{\sigma(\gamma)} h_{k \bar{i}}+\sum h^{a \bar{b}} \partial_{\sigma(\gamma)} h_{k \bar{b}} \partial_{\sigma \theta} h_{a \bar{i}}\right)\right] d z^{\alpha} \wedge d z^{\beta} \wedge d \bar{z}^{\gamma} \wedge d \bar{z}^{\theta} .
\end{aligned}
$$

\section{Curvature of $E \rightarrow \mathcal{C}$}

Let $\varphi: \mathcal{C} \rightarrow C$ be a fibration of curves over a curve. Then we can form an induced connection $D$ bearing it from $\left.E\right|_{C}$ and every curve $\left.E\right|_{\varphi^{-1}(c)}, c \in \mathcal{C}$.

Thus we can differentiate a section of a bundle $E$ using connection $D$ induced from the flat connections from both directions. In this case the action of a connection $D$ along $\left.E\right|_{C}$ will be zero, but the action of $D$ along the other direction may not be zero. We can again consider a matrix of the second derivatives of the metric $h_{i} \bar{j}$. Let us denote the basis of a local frame field as $s_{U}=\left(s_{1}, \ldots, s_{r}, s_{1}^{\prime}, \ldots, s_{l}^{\prime}\right)$, where $s=\left(s_{1}, \ldots, s_{r}\right)$ is a frame field along $\left.E\right|_{\varphi^{-1}(c)}$ and $s^{\prime}=\left(s_{1}^{\prime}, \ldots, s_{l}^{\prime}\right)$ is a local frame field along $\left.E\right|_{C}$. Thus

$$
D(s)=\nabla_{\varphi^{-1}(c)}(s)+\nabla_{C}\left(s^{\prime}\right) .
$$

Therefore, when we form a corresponding matrix of an action of connection $D$ on the local frame section, it is $D(s)=\left(\nabla_{\varphi^{-1}(c)}+\nabla_{C}\right)\left(s_{1}, \ldots, s_{r}, s_{1}^{\prime}, \ldots, s_{r}^{\prime}\right)$. 
Let us again consider the coordinates $\left(z_{1}, \bar{z}_{1}, z_{2}, \bar{z}_{2}\right)$. Now the first part of coordinates corresponds to coordinates on the curve $C$ and the second part to those on the curve $\varphi^{-1}(c)$. Thus, locally, we have $s_{U}=\left(s_{1}, \ldots, s_{r}, s_{1}^{\prime}, \ldots, s_{r}^{\prime}\right)$. Denote $h_{i j}=h\left(s_{i}, s_{j}\right), h_{i j}^{\prime}=h\left(s_{i}, s_{j}^{\prime}\right), h_{i j}^{\prime \prime}=h\left(s_{i}^{\prime}, s_{j}^{\prime}\right)$. Notice that $\partial^{2} h_{i j}, \partial^{2} h_{i j}^{\prime}, \partial^{2} h_{i j}^{\prime \prime}$ vanish along $\left(z_{1}, \bar{z}_{1}\right)$ and nowhere else.

Consequently, we will have

$$
\partial^{2}\left(h_{i j}\right)=\left(\begin{array}{cccc}
0 & 0 & \overline{a_{i, i}} & a_{i, j}^{-} \\
0 & 0 & \overline{a_{j, i}} & a_{j, j}^{\bar{j}} \\
a_{i, i} & a_{i, j} & * & * \\
a_{i, j} & a_{j, j} & * & *
\end{array}\right)
$$

\section{Second Chern class for $E \rightarrow \mathcal{C}$}

To analyze a formula for $\Omega^{4}$ we have to understand how the first derivatives $\partial h_{i j}$ involved into the expression

$$
\begin{aligned}
\left(\Omega^{4}\right)_{i i}=\sum_{j} \sum_{\alpha}\left[\sum_{k} \sum_{\sigma \in S_{4}}(-1)^{\sigma}\left(-\partial_{\sigma(\bar{\beta})} \partial_{\sigma(\alpha)} h_{i \bar{k}}+\sum h^{a \bar{b}} \partial_{\sigma(\alpha)} h_{i \bar{b}} \partial_{\sigma \beta} h_{a \bar{k}}\right)\right. \\
\left.\cdot\left(-\partial_{\sigma(\bar{\theta})} \partial_{\sigma(\gamma)} h_{k \bar{j}}+\sum h^{a \bar{b}} \partial_{\sigma(\gamma)} h_{k \bar{b}} \partial_{\sigma \theta} h_{a \bar{j}}\right)\right] \\
\cdot\left[\sum_{k} \sum_{\sigma \in S_{4}}(-1)^{\sigma}\left(-\partial_{\sigma(\bar{\beta})} \partial_{\sigma(\alpha)} h_{j \bar{k}}+\sum h^{a \bar{b}} \partial_{\sigma(\alpha)} h_{j \bar{b}} \partial_{\sigma \beta} h_{a \bar{k}}\right)\right. \\
\left.\cdot\left(-\partial_{\sigma(\bar{\theta})} \partial_{\sigma(\gamma)} h_{k \bar{i}}+\sum h^{a \bar{b}} \partial_{\sigma(\gamma)} h_{k \bar{b}} \partial_{\sigma \theta} h_{a \bar{i}}\right)\right] d z^{\alpha} \wedge d z^{\beta} \wedge d \bar{z}^{\gamma} \wedge d \bar{z}^{\theta}
\end{aligned}
$$

behave in this case. It is clear that $\partial_{z_{1}} h_{i j}=0, \partial_{\overline{z_{1}}} h_{i j}=0$, meanwhile the derivatives from the other direction may not be zero (i.e. $\partial_{z} h_{i j}$ is not zero, $z \in C$ ). If we look at the expression above, it could be deduced that the first derivatives are included in the expression $\sum h^{a \bar{b}} \partial_{\partial_{i}} h_{k \bar{b}} \partial_{z_{j}} h_{a \bar{i}}$ in pairs (so that at least one of them is always zero as the connection is flat in one direction and hence the first derivative is zero) therefore the terms involving it go to zero and what is left are the only expressions for the second derivatives. If we permute the columns or rows of the matrix, while working with the expression for $\Omega^{4}$, the essential form will stay the same. This way we get the expression

$$
\begin{aligned}
& \kappa= \sum_{i}\left(\Omega^{4}\right)_{i i}=\sum_{i, j, \alpha, \sigma \in S_{4}} \partial_{\sigma(\bar{\beta})} \partial_{\sigma(\alpha)} h_{i \bar{k}} \cdot \partial_{\sigma(\bar{\beta})} \partial_{\sigma(\alpha)} h_{j \bar{k}} \\
& \cdot \partial_{\sigma(\bar{\theta})} \partial_{\sigma(\gamma)} h_{k \bar{j}} \cdot \partial_{\sigma(\bar{\beta})} \partial_{\sigma(\alpha)} h_{j \bar{k}} d z^{\alpha} \wedge d z^{\beta} \wedge d \bar{z}^{\gamma} \wedge d \bar{z}^{\theta} \\
&=\sum_{i, j} \operatorname{det}\left(\partial^{2} h_{i j}\right) d z^{1} \wedge d \bar{z}^{1} \wedge d z^{2} \wedge d \bar{z}^{2} .
\end{aligned}
$$


If all the expressions with the first derivatives go to zero, we get

$$
\partial^{2}\left(h_{i j}\right)=\left(\begin{array}{cccc}
0 & 0 & a_{\bar{i}, i} & a_{i, j}^{-} \\
0 & 0 & a_{\bar{j}, i} & a_{j, j}^{\bar{j}} \\
a_{i, i} & a_{i, j} & * & * \\
a_{i, j} & a_{j, j} & * & *
\end{array}\right)
$$

We denote by $*$ the elements of derivatives of metric $h_{i j}$ not in the mixed directions. Denote the matrices corresponding to the mixed directions as

$$
D_{i j}=\left(\begin{array}{ll}
a_{i, i} & a_{i, j} \\
a_{i, j} & a_{j, j}
\end{array}\right)
$$

and $\bar{D}_{i j}$ correspondingly. In general, for $E \rightarrow \mathcal{C}, \operatorname{det}\left(\partial^{2}\left(h_{i j}\right)\right)=\operatorname{det}\left(D_{i j}\right) \operatorname{det}\left(\bar{D}_{i j}\right)$ (in the second case the additional minor does not influence the expression of determinant by the Linear Algebra rules).

Theorem 7.1 The resulting formula for $E \rightarrow \mathcal{C}$ is

$$
\begin{aligned}
c_{2}(E) & =\sum_{i}\left(\Omega^{4}\right)_{i i} \\
& =\sum_{i, j} \frac{\operatorname{det}\left(\partial^{2} h_{i j}\right)}{\operatorname{det}\left(h_{i j}\right)} d z^{1} \wedge d \bar{z}^{1} \wedge d z^{2} \wedge d \bar{z}^{2} \\
& =\sum_{i, j} \frac{\operatorname{det}\left(D_{i j}\right) \operatorname{det}\left(\bar{D}_{i j}\right)}{\operatorname{det}\left(h_{i j}\right)} d z^{1} \wedge d \bar{z}^{1} \wedge d z^{2} \wedge d \bar{z}^{2}
\end{aligned}
$$

Thus from the last theorem the stability result follows.

Corollary 7.2 We have for $E \rightarrow \mathcal{C}, c_{2}(E)>0$.

Remark 7.3 Local positivity of $c_{2}$-form holds outside of singular points and in fact, the impact of singular point depends on the dilation (distance on symmetric space $\left.\mathrm{GL}(n, C) / R^{*} U(n C)\right)$, two points corresponding to unitary structures from different branches of the singular curve.

Acknowledgements Elena Lukzen is especially grateful to Jacopo Stoppa for much help and support during her Ph.D. studies. She expresses her gratitude to the Courant Institute of Mathematical Sciences for the kind hospitality during her visits.

Open Access This article is licensed under a Creative Commons Attribution 4.0 International License, which permits use, sharing, adaptation, distribution and reproduction in any medium or format, as long as you give appropriate credit to the original author(s) and the source, provide a link to the Creative Commons licence, and indicate if changes were made. The images or other third party material in this article are included in the article's Creative Commons licence, unless indicated otherwise in a credit line to the material. If material is not included in the article's Creative Commons licence and your intended use is not permitted by statutory regulation or exceeds the permitted use, you will need to obtain permission directly from the copyright holder. To view a copy of this licence, visit http://creativecommons.org/licenses/by/4.0/. 


\section{References}

1. Biswas, I., Hurtubise, J.: Degenerations of bundle moduli (2021). arXiv:2109.13358

2. Biswas, I., Looijenga, E.: Characteristic forms for holomorphic families of local systems (2021). arXiv:2107.10482

3. Bogomolov, F.A.: Holomorphic tensors and vector bundles on projective manifolds. Math. USSR-Izv. 13(3), 499-555 (1979)

4. Bogomolov, F.A.: Stable vector bundles on projective surfaces. Russian Acad. Sci. Sb. Math. 81(2), 397-419 (1995)

5. Bruzzo, U., Peragine, V.: Moduli of rank 2 Higgs sheaves on elliptic surfaces (2021). arXiv:2110.03383

6. Buonerba, F., Bogomolov, F.A.: Dominant classes of projective varieties. Eur. J. Math. 4(4), 1412-1420 (2018)

7. Chen, X., Donaldson, S., Sun, S.: Kähler-Einstein metrics on Fano manifolds. I. J. Amer. Math. Soc. 28(1), 183-197 (2015)

8. Chen, X., Donaldson, S., Sun, S.: Kähler-Einstein metrics on Fano manifolds. II. J. Amer. Math. Soc. 28(1), 199-234 (2015)

9. Chen, X., Donaldson, S., Sun, S.: Kähler-Einstein metrics on Fano manifolds. III. J. Amer. Math. Soc. 28(1), 235-278 (2015)

10. Corlette, K.: Flat $G$-bundles with canonical metrics. J. Differ. Geom. 28(3), 361-382 (1988)

11. Dervan, R., Ross, J.: K-stability for Kähler manifolds. Math. Res. Lett. 24(3), 689-739 (2017)

12. Donaldson, S.K.: Stability of algebraic varieties and Kähler geometry. In: de Fernex, T. et al. (eds.) Algebraic Geometry: Salt Lake City 2015. Proceedings of Symposia in Pure Mathematics, vol. 97.1, pp. 199-221. American Mathematical Society, Providence (2018)

13. Donaldson, S.K.: Anti self-dual Yang-Mills connections over complex algebraic surfaces and stable vector bundles. Proc. London Math. Soc. 50(1), 1-26 (1985)

14. Hitchin, N.: Stable bundles and integrable systems. Duke Math. J. 54(1), 91-114 (1987)

15. Huybrechts, D., Lehn, M.: The Geometry of Moduli Spaces of Sheaves, 2nd edn. Cambridge Mathematical Library. Cambridge University Press, Cambridge (2010)

16. Maruyama, M.: Stable vector bundles on an algebraic surface. Nagoya Math. J. 58, 25-68 (1975)

17. Maruyama, M.: Moduli of stable sheaves. I. J. Math. Kyoto Univ. 17(1), 91-126 (1977)

18. Maruyama, M.: Moduli of stable sheaves. II. J. Math. Kyoto Univ. 18(3), 557-614 (1978)

19. Mumford, D., Fogarty, J., Kirwan, F.: Geometric Invariant Theory. 3rd edn. Ergebnisse der Mathematik und ihrer Grenzgebiete, vol. 34. Springer, Berlin (1994)

20. Mumford, D.: Stability of Projective Varieties. Monographies de L'Enseignement Mathématique, vol. 24, pp. 39-110. L'Enseignement Mathématique, Geneva (1977)

21. Narasimhan, M.S., Seshadri, C.S.: Stable and unitary vector bundles on a compact Riemann surface. Ann. Math. 82, 540-567 (1965)

22. O'Grady, K.G.: Moduli of sheaves on K3's and higher dimensional HK varieties (2021). arXiv:2109.07425

23. Simpson, C.T.: Higgs bundles and local systems. Inst. Hautes Études Sci. Publ. Math. 75(1), 1618-1913 (1992)

24. Uhlenbeck, K., Yau, S.T.: On the existence of Hermitian-Yang-Mills connections in stable vector bundles. Commun. Pure Appl. Math. 39(S), suppl., S257-S293 (1986)

25. Yau, S.T.: On the Ricci curvature of a compact Kähler manifold and the complex Monge-Ampère equation. I. Commun. Pure Appl. Math. 31(3), 339-411 (1978)

Publisher's Note Springer Nature remains neutral with regard to jurisdictional claims in published maps and institutional affiliations. 